\title{
ANÁLISIS FUNCIONAL Y TRATAMIENTO DE UN CASO CON BULIMIA NERVIOSA
}

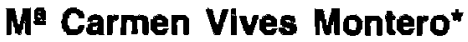 \\ Rafael Ferro García** \\ "Facultad de Psicología. Universidad de Granada \\ "Centro de Psicología Clínica CEDI, Granada
}

\section{RESUMEN}

Este trabajo describe el tratamiento de un trastorno bulímico de una estudiante de bachillerato, con una media de 20 episodios de voracidad semanales seguidos de vómitos autoinducidos. La paciente se manifestaba preocupada por su peso, tenla deseos adelgazar y mostraba ansiedad a los exámenes. Para planificar la intervención se realizó un Análisis Funcional de aquellas situaciones en las que se produclan los episodios bulímicos (situaciones antecedentes), los estímulos que la produclan (el tipo de alimentos ingeridos) y las consecuencias (el escape de situaciones aversivas: las tareas de estudio, la sensación de plenitud excesiva del estómago por la ingesta de alimentos, la ansiedad y la preocupación por ganar peso). El tratamiento se centro: en sustituir las respuestas de escape en las tareas de estudio por otras respuestas altemativas que impedian la interrupción de ésta actividad (vaniar la forma de estudiar); en el entrenamiento de conductas alternativas para

Correspondencia: Me Camen Vives Montero. Departamento de Personalidad, Evaluación y Tratamiento Psicológico. Facultad de Psicologla. Campus Universitario de Cartuja. 18071 Granada (Spain). Teléfono: 958-286650. e-mail: cvives Qugr.es

Deseariamos expresar nuestro agradecimiento a Karen Shashok por la traducción del resumen al inglés. 
reducir la ansiedad (entrenamiento en relajación); en la prevención de episodios de voracidad; y en la exposición con prevención de respuestas a la estimulación que provocaba el vomito autoinducido. Además, se programaron tareas para prevenir las recaídas. Después de cinco meses de tratamiento se eliminaron los problemas bulímicos y se normalizaron los hábitos alimenticios, y la paciente dejó de estar preocupada por la comida y el peso. Durante el año y medio de seguimiento no se volvieron a repetir los episodios bulímicos.

Palabras clave: ANALISIS FUNCIONAL, BULIMIA NERVIOSA, TRATAMIENTO.

\section{SUMMARY}

This article describes the treatment of a female secondary school student with bulimia, manifested as a mean of 20 episodes of binge eating per week followed by self-induced vomiting. The patient was preoccupied about her weight, wished to lose weight and displayed anxiety before school exams. To plan the intervention functional analysis was used to examine situations in which episodes of bulimia occurred (type of food consumed) and the consequences (escape from adversive situations, eg, studying, sensation of a full stomach after eating, anxiety and preoccupation over gaining weight). Treatment centered on: substituting other alternative responses for the escape response to studying (varying the strategies for studying); training alternative behaviors to reduce anxiety (relaxation training); preventing episodes of binge eating; and exposure plus response prevention to stimulation that caused self-induced vomiting. In addition, relapse prevention tasks were scheduled. After five months of treatment, bulimia-related problems had disappeared and eating habits had normalized. The patient was no longer preoccupied about food and her weight. During the one and a half year follow-up period no episodes of bulimia occurred.

Key words: FUNCTONAL ANALYSIS, BULIMIA NERVOSA, TREATMENT. 


\section{INTRODUCCIÓN}

En los últimos años se ha producido un incremento de las investigaciones y publicaciones sobre los trastornos de alimentación. Este interés surge de la necesidad de dar respuesta al incremento de este tipo de trastomos que se está produciendo en la sociedad actual. No es extraño que en una sociedad en la que impera una cultura de la delgadez, potenciada por los medios de comunicación, aumente la preocupación por la figura y la dieta. Pero esta preocupación exagerada llega a hacerse incapacitante para algunas personas y año tras año, se comprueba como se incrementan las consultas por algún trastorno de alimentación (Raich, Mora, Marroquín, Pulido y Soler, 1997; Toro, 1996). Además, la importancia del estudio de estas alteraciones se fundamenta en su inapropiada topografía, en su funcionalidad y en las consecuencias perjudiciales a nivel biológico y conductual que pueden tener para los individuos que la exhiben (Luciano y Molina, 1992).

Los dos tipos de trastomos de la conducta alimentaría principales (anorexia y bulimia) incluidos en el sistema DSM-IV-TR (APA, 2002) comparten dos características fundamentales: la preocupación por el peso y la idea sobrevalorada de adelgazar (Calvo, 1992). Entre estos trastomos del comportamiento alimentario, la bulimia nerviosa ha sido la más estudiada (Saldaña, 2001). Este trastomo se caracteriza por la presencia de atracones o episodios de voracidad, que consisten en la ingesta de alimentos superior a lo normal en un corto periodo de tiempo y la sensación de pérdida de control sobre la ingesta.

Según el DSM-IV-TR (APA, 2002) el diagnóstico de la bulimia nerviosa conlleva tres componentes. El primero hace referencia a la presencia de episodios recurrentes de atracones. El segundo alude a la realización de conductas compensatorias inapropiadas para prevenir el aumento de peso (vómitos autoinducidos; uso de laxantes, diuréticos y/o enemas; ayuno; realizar ejercicio excesivo). Para poder aplicar este diagnóstico, la frecuencia de los atracones y conductas compensatorias debe ser al menos dos veces a la semana durante tres meses. $Y$ el tercer componente, se refiere a la preocupación excesiva por el peso y la silueta. Tal y como indica Saldaña (2001) el procedimiento terapéutico más empleado en el tratamiento de la 
bulimia es el programa cognitivo-conductual derivado del estudio de Fairburn (1981). Posteriormente, varios autores (Fairburn, Marcus y Wilson, 1993; Saldana, 1994; Wilson y Agras, 2002; entre otros) han publicado guías para el tratamiento de la bulimia que son variaciones del programa citado. Básicamente, la intervención consiste en varias fases en las que se emplean técnicas como: uso de auto-registro, modificación de hábitos alimenticios, control de estimulos, entrenamiento en conductas alternativas, reestructuración cognitiva, el mantenimiento de los cambios y la prevención de recaídas.

En algunas investigaciones se ha comprobado que la terapia cognitivo-conductual ha resultado ser más eficaz para el tratamiento de la bulimia que otros tratamientos (Agras, Walsh, Fairburn, Wilson, $y$ Kraemer, 2000; Fairburn, Jones, Peveler, Carr, Solomon, O'Connor, Burton y Hope, 1991; Thackwray, Smith, Bodfish y Meyers, 1993; Whittal, Agras y Gould, 1999; Wilson, Loeb, Walsh, Labouvie, Petkova, Liu y Waternaux, 1999). Sin embargo, estos datos han tenido repercusiones negativas, puesto que a veces se rechaza la aplicación de técnicas conductuales que ya habían demostrado resultados positivos en investigaciones anteriores (Giles, Young y Young, 1985; Jansen, Broekmate y Heymans, 1992; Rosen y Leitenberg, 1982) y se asume con demasiada frecuencia que ante un trastomo bulímico se debe aplicar automáticamente técnicas cognitivo-conductuales. No hay que olvidar que ésta tendencia a asociar un diagnóstico particular con la aplicación de un paquete de tratamiento, ya fue criticada hace tiempo por Barlow (1980). Esta selección automática que realizan algunos terapeutas $\sin$ definir operacionalmente los problemas de conducta de forma previa y sin realizar un análisis funcional, dificulta el diseño de tratamientos más apropiados adaptados a cada caso, llegando incluso a complicar y alargar la intervención más de lo que es necesario.

Por el contrario, últimamente han aparecido publicaciones en las que se pone en evidencia que la terapia cognitivo-conductual no es siempre eficaz con todos las personas que sufren bulimia nerviosa. Toro, Cervera, Feliu, Garriga, Jou, Martínez y Toro (2003) encontraron un grupo de personas con bulimia nerviosa que mostraban resistencia al tratamiento cognitivo-conductual y decidieron tratarlas aplicando técnicas de exposición. Los citados autores consiguieron suprimir los episodios bulímicos de las 6 personas tratadas mediante estas 
técnicas conductuales y comprobaron el mantenimiento de los resultados durante un periodo largo de seguimiento.

Por otra parte, Moreno y Villar (2002) recalcan la existencia de un porcentaje alto de pacientes que pasan por diferentes categorias de los trastornos de alimentación, altemando periodos restrictivos (anorexia) con etapas de sobreingesta y conductas purgatorias (bulimia). Ante estos datos nos planteamos que la clave de estos trastornos alimenticios no radica en la topografía de la conducta problema si no en la función que tienen estas conductas y en las variables que las mantienen. Es decir, estos dos problemas de alimentación pueden ser considerados como conductas equivalentes funcionalmente, puesto que a pesar de tener topografias diferentes comporten la misma función: evitar engordar y/o reducir peso.

En el presente estudio de caso se planteó la necesidad de realizar un Análisis Funcional del trastorno alimenticio que presentaba una chica joven dado que había mostrado ambos tipos de trastornos alimenticios a lo largo del tiempo. Se hipotetizó que los problemas anoréxicos y el trastorno bulímico sufridos antes de la intervención, formaban parte de una clase de respuesta y eran conductas equivalentes funciorialmente. Por esta razón, se partió del supuesto que trabajando con la manifestación actual del problema se resolverían otros problemas de alimentación de forma global. De acuerdo con el análisis funcional realizado se diseñó el tratamiento adaptado a las variables responsables del desarrollo y mantenimiento del trastorno de alimentación.

\section{EXPOSICIÓN DEL CASO}

La paciente (Rosa) era una chica de clase media con 17 años de edad. Rosa vivía con sus padres y sus 3 hermanos en un pueblo (su hermano mayor de 20 años, una hermana de 12 años y otra de 5 años). Acudió a consulta acompañada de sus padres estaban preocupados por el problema de alimentación de su hija. Estaba estudiando el último curso del Bachiller Superior y dedicaba unas dos horas al día a hacer ejercicio físico porque tenía aspiraciones profesionales en el campo del deporte.

Hacía dos años que habia presentado problemas anoréxicos y 
había disminuido drásticamente sus comidas llegando a una reducción de peso de $10 \mathrm{Kg}$ y a la pérdida de la menstruación. Rosa relacionó el inicio del problema con el hecho de que tenía una amiga con anorexia en aquel tiernpo y por su influencia, ella también empezó a obsesionarse por adelgazar a pesar de no encontrarse gorda. En aquel momento no recibió ningún tratamiento ni consejo profesional, pero la paciente relató que al encontrarse demasiado débil hizo un intento por comer más para mejorar su estado de salud y simultáneamente incrementó el ejercicio físico que realizaba para evitar engordar. Poco a poco ella fue superando la fase anoréxica y recuperó su peso normal, pero el problema de alimentación fue transformándose en un trastorno bulímico, apareciendo episodios de voracidad seguidos de vómitos autoinducidos. Este último problema se habia agravado hacia dos meses cuando sus mejores amigas sin saber los motivos empezaron a distanciarse de ella y dejaron de llamarla para salir a pasear y divertirse.

Al iniciar las consultas Rosa pesaba $56 \mathrm{Kg}$ y su estatura era de $1,54 \mathrm{~cm}$. Según informó la paciente los episodios bulímicos solían ocurrir unas 3 ó 4 veces al día. Se sentía angustiada porque sus impulsos por la comida le impedian concentrarse en el estudio. Anteriormente había sido una buena estudiante, pero en la última evaluación le habia suspendido una asignatura por primera vez y no se había presentado a 3 exámenes. Mostraba ansiedad ante los exámenes y conductas de evitación a los mismos. Rosa decía que a veces se encontraba triste y tenía llantos frecuentes que según ella se debían a la frustración que sentía por no poder controlar sus episodios bulímicos.

La paciente solla mantener unas relaciones normales con sus familia según informaron los padres. Ellos la habían considerado una niña responsable hasta ese momento, aunque informaron que se agobiaba mucho ante los problemas y tenía sentimientos de culpabilidad con frecuencia. Dos semanas antes de acudir a consulta, Rosa había comentado a sus padres sus problemas con la comida pero se quedó sorprendida ante la reacción que tuvo el padre, que respondió como si fuese una tragedia sin solución. Su padre se enfadó exageradamente con ella y la amenazó con que él se iba a ir de la casa por culpa de su problema bulímico. Ante este esta 
situación familiar ella se sintió desesperada y tuvo un intento de suicidio con barbitúricos, por lo cual estuvo ingresada en el hospital hasta su recuperación. Después de este acontecimiento Rosa y sus padres se replantearon la forma de abordar el problema y decidieron buscar ayuda profesional. Desde la primera consulta, los padres ponen de manifiesto su disposición para colaborar en el tratamiento y piden consejo sobre cómo ayudar a su hija.

La semana anterior a la primera consulta había roto su relación con un chico. Rosa informó que tenía dificultades para relacionarse con sus amigos y especialmente, para hablar en grupo. Solía negarse a salir con los amigos de su hermano y evitaba ir a las competiciones deportivas. Tenía un buen concepto de sí misma. Cuando se miraba en el espejo no sentía ansiedad ante su imagen. Sin embargo, estaba preocupada por su figura y deseaba reducir su peso. Evitaba ir a celebraciones con sus amigos o con su familia por miedo a comer excesivamente.

Rosa reconocia que tenía un problema y deseaba solucionarlo. Entre los motivos que tenía para querer resolver sus problemas con la comida ella destacó los siguientes: 1. poder incrementar su concentración en el estudio y dejar de perder el tiempo pensando en la comida; 2. ver a sus padres más contentos y tranquilos; 3 . sentirse más fuerte para poder hacer deporte y 4. por mejorar su salud y poder cursar los estudios de Educación Física.

\section{EVALUACIÓN}

Durante las dos primeras consultas se llevó a cabo la evaluación. Para la evaluación del caso se realizaron dos entrevistas clínicas con Rosa y por otro lado, una entrevista con los dos padres. A lo largo de estas entrevistas se recogió la información sobre la historia de la paciente y sus problemas, que fue confirmada y ampliada en las entrevistas con los padres.

Además, se le pidió a Rosa que completara unos autoregistros durante varias semanas en las que debia anotar: todo lo que comía, el número de vómitos autoinducidos, el horario, la situación, la actividad que estaba realizando y las consecuencias. Las anotaciones de los autorregistros confirmó la información recogida en las entrevistas y 
permitió recoger datos adicionales que permitieron realizar el Análisis Funcional.

\section{Análisis Funcionai}

A partir de los registros que la paciente realizó durante 3 semanas antes de iniciar el tratamiento, se pudo confirmar que los episodios bulímicos (seguidos siempre de vómitos autoinducidos) ocurrían de media unas 3 veces al día. Coincidiendo uno por la mañana después de desayunar y otros dos por las tardes a la hora de estudiar (entre las 6 y las 8 de la tarde). Para realizar los episodios de voracidad solía ocultarse de los demás.

Con la información recogida se realizó un Análisis Funcional del que se obtuvieron los siguientes datos:

- Las situaciones en las que se solían producir los episodios de voracidad eran actividades que provocaban estados de ansiedad alta, principalmente estudiar a solas en su cuarto de estudio.

- Los estimulos antecedentes que producían los vómitos autoinducidos eran la ingesta de alimentos hipercalóricos como: dulces, bocadillos, patatas fritas, frutos secos, helados, etc. que le provocaban la consiguiente preocupación por la posibilidad de ganar peso.

- Las consecuencias que estaban manteniendo los episodios de voracidad eran: por un lado, el escape de una situación aversiva (el estudio que era una actividad que le producía ansiedad), y por otro, el reforzamiento positivo automático derivado de la ingestión de comida.

- Las consecuencias que mantenían los vómitos autoinducidos eran: el escape de la sensación de plenitud del estómago y la reducción de la ansiedad ante el temor a ganar peso.

Del análisis de estos datos se dedujo que tanto los episodios de voracidad como los vómitos cumplían fundamentalmente una función de escape de una estimulación aversiva. Estos datos coinciden con las conclusiones de Mora y Raich (1993) que indican que los antecedentes más frecuentes de un episodio bulímico son un humor negativo, en forma de ansiedad, irritabilldad y aburrimiento que se atenúa a medida que transcurre el episodio, emergiendo entonces emociones negativas como culpa o miedo a ganar peso que se intentan paliar mediante el vómito. El alivio durante el vómito se explica por la 
eliminación de la posibilidad de ganancia de peso y de las sensaciones intolerables de la plenitud. Sin embargo, después del vomito suelen producirse sentimientos de vergüenza y frustración.

\section{Diagnóstico}

Con la información recogida durante la evaluación, se confirmó que cumplía los tres criterios que incluye el DSM-IV (APA, 2002) para el diagnóstico de bulimia nerviosa. Presentaba atracones de comida recurrentes; realizaba conductas compensatorias para prevenir la ganancia de peso (vómitos autoinducidos y ejercicio físico), y se mostraba preocupada por su peso.

\section{TRATAMIENTO}

Los objetivos de tratamiento que se plantearon fueron: 1. normalizar el horario y cantidad de comidas ingeridas; 2. enseñar formas alterativas de manejar la ansiedad; 3. eliminar sus respuestas de escape (episodios bulímicos).

La intervención incluyó diferentes componentes entre los que destacan:

- la modificación de los hábitos alimenticios;

- el entrenamiento en conductas altemativas;

- manejo de contingencias por parte de la familia;

- la exposición a las situaciones de riesgo y la prevención de respuestas;

- la adquisición de conductas para afrontar la ansiedad (relajación) junto con las técnicas de control de estímulo y

- la planificación de la prevención de las recaídas.

Para la aplicación del tratamiento se requirieron 13 sesiones distribuidas a lo largo de 5 meses. Durante todo el tratamiento, Rosa continuó realizando el auto-registro descrito anteriormente. Posteriormente se realizó una fase de 3 sesiones de revisión (cada 3 semanas). Por último, hubo una fase de seguimiento con una sesión mensual durante seis meses y un año después de haber finalizado las consultas se obtuvo información telefónica de los resultados. Todas las consultas se dedicaron a trabajar directamente con Rosa 
la mayor parte del tiempo y otra parte de la sesión se dedicaba a intercambiar información con los padres (que siempre la acompañaban a consulta).

En la primera sesión de tratamiento se explicaron los datos y las conclusiones obtenidas de la evaluación. Se comenzó a intervenir modificando sus hábitos alimentarios. Para esto se le dieron a Rosa y a la madre las siguientes indicaciones:

- debía comer exclusivamente en la cocina;

- no tenía que realizar otras actividades de forma concurrente con la ingestión de comida;

- debía planificar con antelación la menienda y reducir la cantidad de dulces que ingería;

- debía dejar transcurrir al menos dos horas entre comidas para volver a comer.

Para enseñar a la paciente formas alternativas de manejar la ansiedad, se inició el entrenamiento en relajación según el procedimiento de Jacobson (1938). Por otro lado, se les aconsejó a los padres que no acumularan alimentos preparados ni dulces, galletas, etc. y que estuviesen pendiente de ella y no la dejasen sola en la casa en las primeras horas de la tarde que era el momento de mayor riesgo para las conductas bulímicas.

En la segunda y tercera sesión se comprobaron las tareas programadas en la sesión anterior y se reforzo su cumplimiento. Se continuó con el entrenamiento en relajación. Estas sesiones se centraron en reducir las respuestas de escape de las tareas de estudio. Se le pidió que modificara los horarios del estudio, comenzando por establecer periodos cortos de estudio con descansos para hacer otras actividades distintas y de forma paulatina, se incrementó el periodo de estudio. Se hicieron ensayos encubiertos en los que Rosa se imaginaba que impedia las interrupciones durante el estudio prosiguiendo con la misma actividad, pero realizando otras conductas alternativas que consistian en variar la forma de estudiar. Es decir, cuando ella empezaba a sentir deseos de ir a comer algo sin tener hambre, cambiaba la tarea de estudio que estaba realizando por otras técnicas de estudio más activas como realizar esquemas, resúmenes y/o repasar lo estudiado. Al finalizar la sesión se le pidió que pusiera en práctica durante la semana este tipo de ensayos. A los padres se les 
dieron instrucciones para que extinguieran los comentarios de Rosa en relación con la comida o su miedo a engordary que reforzaran los cambios que le observaran.

En la cuarta sesión y quinta sesión se comprobaron las tareas y de nuevo se reforzó el cumplimiento de las mismas y se continuó con el entrenamiento en relajación. Estas dos sesiones se dedicaron a la prevención de los episodios de voracidad. Para esto se realizaron ensayos encubiertos en los que ella se imaginaba que comenzaba a sentir impulsos de comer algo fuera de la hora y a continuación, realizaba otras actividades incompatibles, como pasear, charlar con alguien, hacer deporte, etc. Adicionalmente, para interrumpir las cadenas de comida descontrolada sin hambre, se aplicó las habilidades de relajación aprendidas para reducir la ansiedad, así como, realizar otras actividades alternativas. Posteriormente se le pidió que pusiera en práctica estos ensayos durante la semana cuando se dieran esas circunstancias.

En la sexta sesión de tratamiento se comprobó que se habían reducido los episodios de voracidad durante el estudio, pero seguían produciéndose durante la merienda los episodios bulímicos. Ante esto se le recomendó la aplicación de la relajación a la hora de la merienda, que preparara lo que iba a merendar con antelación y cuando terminase se saliera a la calle a visitar a algún familiar. En esta sesión se inició la exposición en imaginación a las sensaciones de plenitud de estómago que eran los estímulos antecedentes de los vómitos autoinducidos. Durante la sesión se realizaron ensayos encubiertos en los que Rosa se imaginaba a sI misma sintiendo su estómago demasiado lleno y con deseos de vomitar, pero entonces se le pedía que aplicase la relajación y que realizara alguna actividad incompatible (hablar con algún familiar, salir a dar un paseo, etc.). Se le dijo que tenfa que poner en práctica estos ensayos durante la semana contando con la supervisión y el reforzamiento de sus progresos por parte de la familia.

La séptima sesión se prosiguió con el entrenamiento en relajación y se inicio la exposición a las sensaciones de plenitud de estómago después de haber comido alimentos de alto valor calórico (que era un desencadenante de vómitos autoinducido). En estas situaciones se le requirió que aplicase la relajación y que realizara otras actividades como salir a pasear, ir a visitar a algún familiar, etc., contando con la 
colaboración de la familia.

En la octava y novena sesión se comprobó que los episodios de voracidad se estaban reduciendo pero los vómitos seguían produciéndose. En estas sesiones se resolvieron los problemas que se iban planteando respecto a la aplicación del tratamiento y se reforzó el cumplimiento de las tareas programadas. La terapeuta se planteó comenzar la intervención en habilidades sociales pero al comprobar que Rosa espontáneamente habla cambiado su círculo de amigos y había comenzado a relacionarse con los amigos de su hermano que hasta entonces solía evitar, se decidió posponer esta intervención y esperar a ver la evolución que ella sola había iniciado.

En la décima sesión se aplicó la exposición con prevención de respuestas. Para lo cual, se le requirió a ella junto con sus padres que viniese a consulta inmediatamente después de que Rosa se hubiese dado un atracón de comida (dulces) y con la sensación de plenitud de estómago. De esta manera se pudo comprobar la ausencia de vómitos autoinducidos a lo largo de la consulta y se garantizó que Rosa soportaba este tipo estimulación. Una vez superada esta situación de alto riesgo se le pidió a los padres que volvieran a acumular en casa alimentos preparados, dulces, galletas, etc. y que comprobaran que Rosa no se excedía comiéndolos.

En la undécima sesión se detectó que la exposición con prevención de respuestas realizada en la sesión anterior había sido efectiva y en los registros semanales se produjo una reducción drástica de los episodios bulímicos. La mejoría fue confirmada por sus padres y reforzada apropiadamente. Se repitieron las técnicas de exposición en imaginación de las situaciones de alto riesgo seguidas de la imaginación de respuestas alternativas y la aplicación de relajación. Se dejó de intervenir en el estudio porque Rosa había terminado el bachiller y decidió no seguir estudiando por el momento. En esta sesión se les pidió a los padres que comenzaran a dejarla sola en la casa en las primeras horas de la tarde (momento de mayor riesgo para las conductas bulímicas) y que comprobaran la comida que ingería en su ausencia.

En la duodécima sesión de nuevo se le requirió que viniese a consulta inmediatamente después de un atracón. Se reforzaron los progresos conseguidos, puesto que estaba controlando mejor las cantidades de alimento que tomaba en las meriendas y estaba 
reduciendo los vómitos autoinducidos. Sus comidas y la distribución temporal de las mismas se estaban normalizando, tal y como cónfirmó su madre. Rosa se comprometió a aplicar la relajación para disminuir su ansiedad antes de las comidas y ante pensamientos o deseos de comer fuera de horas. Paralelamente, la madre y ella informaron que salía con más frecuencia con los amigos y amigas del hermano y que sus dificultades para relacionarse con ellos se estaban reduciendo. Cada vez estaba participando más en las conversaciones en grupo y pasaba un rato agradable y divertido cuando salía con ellos.

En la decimotercera sesión se comprobó que se habían eliminado los episodios bulímicos durante las tres semanas que transcurrieron desde la última sesión. Se reforzaron los progresos y se requirió a la paciente que verbalizara qué estaba haciendo ella para conseguir tan buenos resultados. Durante esta sesión Rosa comentó que estaba incrementado las relaciones con sus amigos y que se sentía más integrada en el grupo. Además, afirmó que estaba contenta y más estable emocionalmente, a pesar de no haber llegado a intervenir directamente sobre estos objetivos.

Ante la ausencia de episodios bulímicos en las últimas semanas se planteó pasar a la fase de seguimiento, pero como faltaban menos de dos meses para Navidad y había un alto riesgo de recaídas por las comidas y celebraciones propias de ésta época, se decidió pasar por una fase de revisión con sesiones cada 3 semanas para garantizar el mantenimiento de los resultados. Después de tres sesiones de revisión, en Enero se comprobó que los resultados se hablan mantenido y se decidió continuar con una fase de seguimiento con una sesión mensual hasta el mes de Junio, es decir, durante seis meses más. Esta decisión de desvanecer las sesiones terapéuticas se tomó al comprobar que Rosa no comía entre comidas y tomaba cantidades normales de los alimentos que antes solía consumir de forma abusiva.

\section{RESULTADOS}

Como se puede comprobar en la Figura 1, durante las 3 semanas que se tomaron datos de Línea Base la frecuencia de los episodios bulímicos oscilaron entre 19 y 22 episodios semanales.

A partir de la tercera sesión de tratamiento se produjo una 


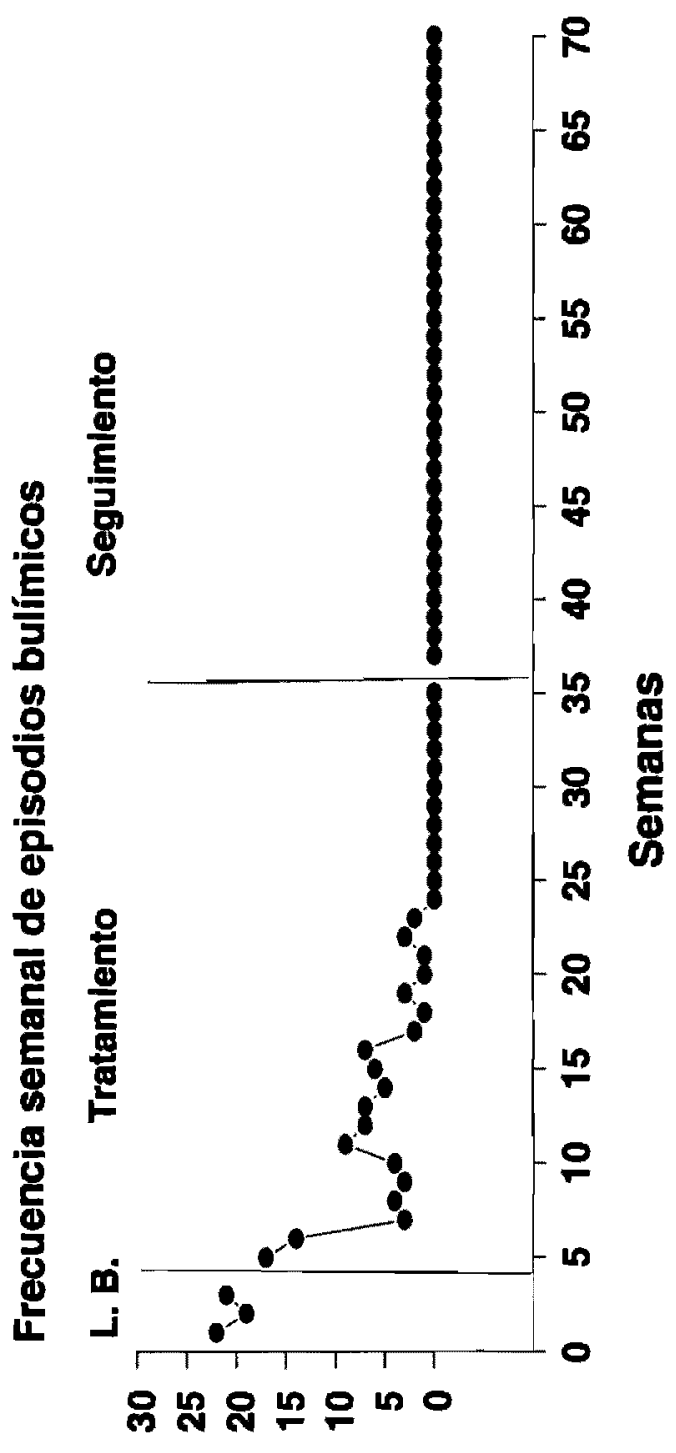

solposjda ap \$N 
disminución drástica de los episodios bulímicos, puesto que la frecuencia semanal se redujo a menos de 5 episodios semanales. Después de ésta reducción de episodios bulímicos, un mes después se produjo un leve incremento a 9 episodios semanales, coincidiendo con las vacaciones en el mes de Agosto, durante el cual se redujeron sus actividades cotidianas. A partir de la duodécima sesión (semana 23) se eliminaron totalmente los episodios bulímicos y Rosa llegó a ser capaz de comer alimentos de alto riesgo (dulces, etc.) sin provocarse los vómitos. Poco después dejaron de aparecer los llantos que presentaba con frecuencia, y manifestó sentirse más contenta. Además, la familia describió que estaba más estable emocionalmente.

En la fase de seguimiento no volvieron a producirse recaidas y a partir de la semana 50, Rosa manifestó las siguientes mejorías:

- Habla perdido su preocupación por la comida y por perder peso.

- Comía dulces y alimentos hipercalóricos sin sentir ansiedad, ni deseos de vomitar.

- Cuando la ocasión lo requería (celebraciones y acontecimientos familiares) se había excedido un poco en la comida sin perder el control y sin provocarse vómitos.

- Se encontraba muy integrada en el grupo de amigos que tenía. Se relacionaba mejor con ellos y salía con más frecuencia.

- Se sentía más contenta y las alteraciones en su estado de ánimo habian desaparecido.

Después de 6 meses sin recaídas se dió por finalizado el periodo de seguimiento y se eliminaron los registros. Un año después, se obtuvo información telefónica de la paciente confirmando que se hablan mantenido estos resultados durante el año transcurrido.

\section{CONCLUSIONES}

A la vista de los resultados, se puede concluir que el Análisis Funcional y la intervención que se llevó a cabo fue efectiva para resolver el trastorno bulímico expuesto. La historia de la paciente con un trastorno anoréxico previo y el intento de suicidio que requirió su hospitalización, indica la gravedad del problema. Después de año y medio de seguimiento se comprobó que no volvieron a producirse ni trastornos bulímicos ni anoréxicos con lo cual se puede dar por 
confirmada la hipótesis de que arnbos formaban parte de una clase de respuesta y eran equivalentes funcionalmente, cuya función era: evitar engordar, adelgazar y reducir su miedo a engordar.

El Análisis Funcional realizado en base a los auto-registros de la paciente, permitió detectar tanto las variables antecedentes como las consecuentes que mantenían el trastomo. La intervención de este caso estuvo basada en la modificación de los hábitos alimenticios, el entrenamiento en conductas alternativas, manejo de contingencias por parte de la familia, en la exposición a las situaciones de riesgo y la prevención de respuestas, la adquisición de conductas para afrontar la ansiedad (relajación) junto con las técnicas de control de estímulos. De igual modo, la planificación de la prevención de las recaídas fue otro elemento importante en la intervención para producir estos resultados.

Desde el comienzo del tratamiento hubo una reducción de los episodios de voracidad y de los vómitos autoinducidos. Durante las vacaciones de verano se produjo una leve recaída que coincidió con la reducción de sesiones terapéuticas y a la disminución de sus ocupaciones (su gimnasio cerró por vacaciones, algunos amigos se fueron de vacaciones, etc.). Al finalizar la intervención se comprobó una mejoría tanto de sus hábitos de alimentación como de sus relaciones sociales y su estado emocional aunque estos dos últimos ámbitos no habían sido tratados directamente. El desvanecimiento de las sesiones y de los procedimientos durante la fase de seguimiento promocionó el mantenimiento de los resultados durante un año y medio.

Habría que señalar que tanto la selección de los objetivos como de los procedimientos terapéuticos utilizados, se basaron en el Análisis Funcional realizado. Tal y como afirma Pérez Álvarez (1996) el Análisis Funcional ofrece una explicación pluricausal, contextual y dinámica del funcionamiento psicológico. En el caso expuesto se aplicó una intervención idiosincrática, ya que se adaptó a las variables responsables del desarrollo y mantenimiento de los problemas de ésta cliente. Esta intervención ha tenido en cuenta los objetivos que el Análisis Funcional debe cumplir según Follette, Naugle y Linnerooth (2000) consistentes en: identificar la conductas objetivo y las condiciones que las mantienen, seleccionar una intervención apropiada y ayudar a evaluar y a controlar la efectividad de esa intervención. 
Paralelamente a la aplicación del tratamiento conductual se produjeron cambios positivos en el ámbito social y emocional, así como la reducción de la preocupación de la paciente sobre su peso sin haber sido tratadas directamente. Este tipo de mejorlas ya fueron puestas de manifiesto en la revisión realizada por Rosen (1987) que afirmó que la mayoria de los estudios que revisó demostraban que los pacientes tratados con técnicas conductuales focalizados en la modificación de los episodios de voracidad y vómitos, también manifestaban mejorias en sus relaciones sociales, imagen corporal y estados emocionales, en contra de las criticas que han recibido estos tratamientos. Algunos autores como Thackwray, Smith, Bodfish y Meyer (1993), defienden que la bulimia nerviosa debe ser tratada con tratamientos multicomponentes que intervengan directamente sobre los pensamientos distorsionados, las conductas problemáticas y sobre el desarrollo de habilidades de afrontamiento. Sin embargo, en investigaciones anteriores (Giles, Young y Young, 1985; Jansen, Broekmate y Heymans, 1992; Rosen y Leitenberg, 1982) se recogen datos que demuestran que los tratamientos conductuales también producen mejorías en diversos componentes del trastorno aunque no hayan sido objetivo directo del tratamiento. Los resultados del presente estudio nos lleva a plantear la duda sobre la necesidad de la aplicación de los tratamientos cognitivo-conductuales para que produzcan cambios en todos los componentes del trastorno bulímico en todos los casos de bulimia nerviosa.

\section{REFERENCIAS BIBLIOGRÁFICAS}

Agras, W.S., Walsh, T., Fairburn, C.G. Wilson, G.T. y Kraemer H.C. (2000). A multi-center comparison of cognitive-behavioral therapy and interpersonal psychotherapy for bulimia nervosa. Archives of General Psychiatry, 57, 459-466.

American Psychatric Association (2002). DSM-IV-TR. Manual de Diagnóstico y Estadistico de los Trastornos Mentales. Barcelona: Masson.

Barlow, D. (1980). Behavior therapy: the next decade. Behavior Therapy, 11, 315-328.

Calvo, R. (1992). Trastornos de la alimentación (I). En E. Echeburúa 
(Ed.). Avances en el tratamiento psicológico de los trastornos de ansiedad. Madrid: Pirámide.

Fairburn, C.G. (1981). A cognitive behavioral approach to the management of bulimia. Psychological Medicine, 11, 707-711.

Fairburn, C.G. Jones, R., Peveler, R.C., Carr, S.J., Solomon R.A., O'Connor, M.E., Burton, J. y Hope, R.A. (1991). Three psychologica treatments for bulimia nervosa: A comparative trial. Archives of General Psychiatry, 48, 463-469.

Fairburn, C.G. Marcus, M. D. y Wilson, G. (1993). Cognitive behavioral therapy for binge eating and bulimia nervosa: $A$ comprehensive treatment manual. En C.G. Fairburn y G.T. Wilson (Eds). Binge eating: nature, assessment and treatment. (pp. 361404). New York: Guilford Press.

Follette, W.C., Naugle, A.E. y Linnerooth, P.J.N. (2000). Functional alternatives to traditional assessment and diagnosis. En M.J. Dougher (ed.). Clinical Behavior Analysis. (pp. 99-125). Reno: Context Press.

Giles, T.R., Young, R.R. y Young, D.E. (1985). Behavioural treatment of severe bulimia. Behavior Therapy, 16, 393-405.

Jacobson, E. (1938). Progressive relaxation. Chicago: University of Chicago Press.

Jansen, A., Broekmate, J. y Heymans, M. (1992) Cue exposure vs. self-control in the treatment of binge eating: A pilot study. Behaviour, Research and Therapy, 3, 235-241.

Luciano, M.C. y Molina, F: (1992). Rumiación y vómitos: etiología, prevención y tratamiento. Análisis y Modificación de Conducta, 18, 58, 255-277.

Mora, M. y Raich, R.M. (1993). Sintomatología bulímica: un análisis topográfico y funcional. Análisis y Modificación de Conducta, 19, 66, 479-509.

Moreno, S. y Villar, S. (2002). Características clínicas y tratamiento de los trastomos de la conducta alimentaria. En V. Caballo y M.A. Simón (Eds). Manual de Psicología Clinica Infantil y del Adolescente. (pp. 261-289). Madrid: Pirámide.

Pérez Álvarez, M. (1996). Tratamientos psicológicos. Madrid: Universitas, S.A.

Raich, R.M., Mora, M., Marroquin, H., Pulido, S.A. y Soler, A. (1997). 
Tratamiento Cognitivo-Conductual de la insatisfacción corporal. Análisis y Modificación de Conducta, 23, 89, 403-424.

Rosen, J.C. (1987). A Review of Behavioral Treatments for Bulimia Nervosa. Behavior Modification, 11, 464-486.

Rosen, J.C. y Leitenberg, H. (1982). Bulimia nervosa: treatment with explosure and prevention. Behavior Therapy, 13, 117-124.

Saldaña, C. (1994). Trastomos del comportamiento alimentario. Madrid: Fundación Universidad Empresa.

Saldaña, C. (2001). Tratamiento psicológicos eficaces para los trastomos del comportamiento alimentario. Psicothema, 13. 381-392.

Thackwray, D. E., Smith, M.C., Bodfish, J. W. y Meyers, A. W. (1993). A Comparison of Behavioral and Cognitive-Behavioral Interventions for Bulimia Nervosa. Joumal of Consulting and Clinical Psychology, 61, 639-645.

Toro, J. (1996). El cuerpo como delito. Barcelona: Ariel.

Toro, J., Cervera, M., Feliu, M.H., Garriga, N., Jou, M., Martínez, E.

y Toro, E. (2003). Cue Exposure in the Treatment of Resistant Bulimia Nervosa. International Joumal of Eating -Disorders, 34, 227-234.

Whittal, M.L., Agras, W.S. y Gould, R.A. (1999). Bulimia nervosa: A meta-analysis of psychological and pharmacological treatment. Behavior Therapy, 30, 117-135.

Wilson G.T. y Agras, W.S. (2002). Practice guidelines for eating disorders. Behavior Therapy, 32, 219-234.

Wilson, G T., Loeb, K.L., Walsh, B.T., Labouvie, E., Petkova,E., Liu, $X$ y Waternaux, C. (1999). Psychological versus pharmacological treatment of bulimia nervosa: Predictors an processes of change. Joumal of Consulting and Clinical Psychology, 67, 451-459. 